\title{
Tai chi in patients with heart failure with preserved ejection fraction
}

Gloria Y. Yeh, MD MPH ${ }^{1,2}$, Malissa J. Wood, MD ${ }^{3}$, Peter M. Wayne, PhD ${ }^{1,4}$, Mary T. Quilty, BS $^{1,4}$, Lynne W. Stevenson, MD ${ }^{5}$, Roger B. Davis, ScD ${ }^{1,2}$, Russell S. Phillips, MD ${ }^{1,2}$, and Daniel E. Forman, MD ${ }^{5,6}$

${ }^{1}$ Osher Center for Integrative Medicine, Harvard Medical School

${ }^{2}$ Division of General Medicine and Primary Care, Department of Medicine, Beth Israel Deaconess Medical Center

${ }^{3}$ Division of Cardiology; Massachusetts General Hospital

${ }^{4}$ Division of Preventive Medicine; Brigham and Women's Hospital

${ }^{5}$ Cardiovascular Division; Brigham and Women's Hospital

${ }^{6}$ New England Geriatric Research, Education, and Clinical Center, Veterans Administration Boston Healthcare System

\section{Abstract}

Background-Although exercise is an important component of heart failure management, optimal regimens, particularly in heart failure with preserved ejection fraction (HFPEF), are uncertain. Tai chi (TC) is a mind-body exercise that may have potential benefits but has not been studied in this population.

\begin{abstract}
Methods-We randomized sixteen patients with HFPEF to either 12 weeks TC or aerobic exercise . Assessments included peak oxygen uptake, 6-minute walk, quality-of-life, echocardiography, mood, and self-efficacy at baseline and 12 weeks. Cardiorespiratory measures during exercise were obtained to characterize training intensities.
\end{abstract}

\begin{abstract}
Results-Baseline characteristics were as follows: age $66 \pm 12$ years, E/A ratio $1.3 \pm 0.7$, E/e' ratio $15.9 \pm 4.8$. Adherence overall was excellent ( $89 \%$ attendance). Change in peak oxygen uptake was similar between groups after 12 -weeks, but 6-minute walk distance increased more after TC $(+69 \pm 46 \mathrm{~m}$ vs. $+10 \pm 31 \mathrm{~m}, \mathrm{p}=0.02)$. While both groups improved Minnesota-Living-with-HeartFailure score and self-efficacy, POMS-depression scores improved more with TC $(-1.7 \pm 2.8$ vs. $+1.6 \pm 3, \mathrm{p}=0.05)$. Cardiorespiratory assessment during TC showed lower oxygen uptake $(4.3 \mathrm{ml} /$ $\mathrm{kg} / \mathrm{min}$ vs. $9.4 \mathrm{ml} / \mathrm{kg} / \mathrm{min}, \mathrm{p}<0.01$ ), respiratory rate and heart rate
\end{abstract}

Conclusions-TC is feasible and safe in HFPEF. Therepeutic endpoints appear similar with TC relative to aerobic exercise despite a lower aerobic training workload.

\section{Introduction}

Heart failure with preserved ejection fraction (HFPEF) is widely prevalent and growing in incidence, particularly since aging increases susceptibility to the disease and the population of elderly is expanding. ${ }^{1}$ Definitive therapy for HFPEF, however, remains unclear and there is a critical need to expand and optimize therapeutic insights and options.

Please address correspondence to: Gloria Y. Yeh, MD, MPH Division of General Medicine and Primary Care Beth Israel Deaconess Medical Center 1309 Beacon Street Brookline, MA 02446 gyeh@hms.harvard.edu office: 617-754-1419 fax: 617-754-1440. 
Exercise training is an important component of heart failure management, however most studies to date have investigated heart failure with systolic dysfunction. More recently, exercise training in HFPEF has gained attention. One study by Kitzman et al demonstrated that a 16-week aerobic training regimen provided significant benefits in HFPEF. ${ }^{2}$ Peak exercise oxygen consumption and 6-minute walk distance improved significantly as compared to an attention control group. Physical quality of life score also improved $(\mathrm{P}=0.03)$. These data provide important rationale for exercise. Unfortunately, multiple trials have demonstrated problematic adherence to aerobic exercise over time, particularly with diverse populations and settings. ${ }^{3-4}$ Moreover, studies have suggested that peripheral effects play a particularly important role. ${ }^{5}$ Thus, one question that has been raised is whether exercise gains might be adequately and efficiently achieved with alternative training options that may directly address these behavioral and physiological issues.

Tai chi (tai chi chuan or taijiquan) is a gentle, meditative exercise that has its roots in ancient Chinese martial arts. It employs detailed regimens of flowing circular arm movements, balance and weight shifting, breathing techniques, and cognitive tools (such as visualization and focused internal awareness). ${ }^{6-7}$ Also inherent in tai chi is a component of core strengthening and stretching. This may facilitate a beneficial training effect particularly in those who are deconditioned, older, or have chronic disease. In addition, compared to aerobic training, the relatively lower intensity and meditative qualities of tai chi may help accessibility and promote longer-term exercise adherence over time. Prior studies have reported good long term adherence with tai chi in various chronic disease populations. ${ }^{8-11}$

In prior studies, we investigated the potential of tai chi to improve exercise capacity and quality-of-life in patient with systolic heart failure. ${ }^{12-13}$ In this pilot investigation, we expand our inquiry to explore the potential benefits of tai chi for HFPEF. Our aims were to preliminarily assess changes in functional and physiological outcome measures after 12 weeks of tai chi as compared to low intensity aerobic exercise, explore relative differences in training intensities, and determine feasibility of a larger, more definitive trial.

\section{Methods}

\section{Study design}

Sixteen patients were recruited from the ambulatory cardiology practice at Beth Israel Deaconess Medical Center in Boston, MA. Inclusion criteria were based on the following: 1) physician diagnosis of HFPEF; 2) New York Heart Association functional class I, II or III; 3) left ventricular ejection fraction $\geq 50 \%$ (by echocardiography, radionuclide angiography or contrast angiography) within 2 years of screening. Exclusion criteria included: 1) unstable angina, myocardial infarction or major cardiac surgery in the past 3 months; 2) cardiac arrest in the past 6 months; 3 ) significant valvular or pericardial disease accounting for signs and symptoms of heart failure; 4) severe chronic obstructive pulmonary disease on bronchodilators or chronic lung disease with cor pulmonale; 5) moderate or severe pulmonary hypertension; 6) atrial fibrillation as the dominant rhythm; 7) severe peripheral vascular disease, claudication or other physical condition that would preclude a walk test ;8) inability to perform bicycle ergometry; 9) technically inadequate echocardiographic windows or valvular conditions precluding assessment of diastolic function, ie. patients with severe mitral annular calcification; 10) cognitive dysfunction (MMSE $\leq 24), 11$ ) nonEnglish speaking; 12) current participation in conventional cardiac rehabilitation or regular practice of tai chi.

Patients were assigned randomly to receive 12 weeks of either tai chi or aerobic exercise. All participants continued to receive their usual care which included pharmacologic therapy and general exercise advice as per the ACC/AHA HF guidelines. Permuted block 
randomization with variable block size was used to generate treatment assignments. All patients provided written informed consent. The Beth Israel Deaconess Medical Center's human subjects review board approved the protocol.

\section{Tai chi intervention}

The tai chi intervention consisted of one-hour group classes held twice weekly for 12 weeks with an experienced instructor. A standard training regimen was utilized that we have used in prior trials for patients with systolic heart failure (Table 1). The protocol included traditional warm-up exercises followed by five simplified tai chi movements. Classes incorporated weight shifting, arm swinging, gentle stretches (of the neck, shoulders, spine, arms, legs), visualization techniques, and traditional breathing. These exercises focus on releasing tension in the physical body, incorporating mindfulness and imagery into movement, increasing awareness of breathing and promoting overall relaxation of body and mind. The core tai chi movements were adapted from Master Cheng Man-Ch'ing's Yangstyle short form ${ }^{6}$ and performed repetitively. Chairs were provided for resting, and patients were allowed to progress at their own comfort and pace.

Each subject in the tai chi group was also provided with a 35-minute instructional videotape that reviewed the exercises presented in class and was encouraged to practice at home at least three times per week.

\section{Aerobic exercise control}

The conventional aerobic exercise group also met for one hour twice a week under the guidance of an experienced instructor. The aerobic training was modeled on a typical community-based conventional low-impact aerobic exercise class offered at a local community center and designed for a senior population with low to moderate training intensity. Warm-up, peak exercise, and cool-down stages were routine. Exercises included stretching of the torso/waist, neck, upper and lower extremity; step in place and side to side with rhythmic leg and arm movements, and seated strengthening exercises. One or two pound hand weights and resistance bands were optional. Chairs were available for seated cool-down exercises and for support when performing basic stretches and strengthening exercises. As this class replicated what was readily available in the community, patients were allowed to progress at their own comfort and pace.

Each subject in the aerobic exercise group was also provided with a 35-minute instructional videotape that reviewed the exercises and instructions presented in class, and subjects were encouraged to practice at home at least three times a week.

\section{Outcome measures}

Comprehensive assessments were completed at baseline and 12 weeks.

Exercise capacity and functional status-Symptom-limited cardiopulmonary exercise testing was completed using an electronically calibrated upright bicycle and continuous electrocardiographic monitoring. A standard 10 watt graded bicycle ramp protocol was utilized. ${ }^{41}$ Patients were encouraged to exercise to exhaustion. Gas exchange was assessed using a SensorMedics (Yorba Linda, CA) metabolic cart. Peak oxygen uptake $\left(\mathrm{VO}_{2}\right)$ values were averaged from the final 20 seconds of the test. The exercise testing staff was blinded to subjects' exercise group assignments.

The six-minute walk test (6MW) per the American Thoracic Society protocol and timed upand-go (TUG) assessments were also completed. ${ }^{14-16}$ The $6 \mathrm{MW}$ is often cited as providing a more meaningful assessment of functional performance with respect to daily activity. TUG 
serves as an integrative assessment of functional and strength capacities and is often used to gauge susceptibility to falls. The staff assessing 6MW and TUG was blinded to the subjects' exercise group assignments.

Physical activity was assessed using the Community Healthy Activities Model Program for Seniors (CHAMPS) Physical Activity Questionnaire for Older Adults, ${ }^{17}$ an assessment which captures weekly frequency and total time spent in different activities and allows estimation of caloric expenditure.

Health-related quality-of-life, symptoms, mood, psychosocial functioningThe Minnesota Living with Heart Failure Questionnaire (MLHF) was used to assess healthrelated quality-of-life. MLHF consists of 21 validated items covering physical, psychological, and socioeconomic dimensions (e.g. swelling in the ankles, difficulty climbing stairs, fatigue, feeling depressed, spending money for heart failure). The score range is 0 to 105 , with a lower number denoting better quality-of-life. ${ }^{18}$

The Profile of Mood States (POMS) is a well-validated instrument for assessing emotional states that are transient and expected to respond to clinical intervention. ${ }^{19}$ It has been widely used to assess the effects of exercise interventions. We used the POMS-Brief version which consists of 30 single-word items rated on a 5-point scale to indicate recent mood in 6 dimensions: tension/anxiety, depression/dejection, anger/hostility, vigor/activity, fatigue/ inertia, and confusion/bewilderment. A decreased total mood disturbance score denotes an improved emotional state (range 0-200). Prior studies of tai chi have reported improvement in mood, decrease in anxiety, and enhancement in vigor as measured by the POMS scale. ${ }^{20}$

The Self-Efficacy-Barriers to Exercise Scale is a reliable and validated measure which assesses one's confidence to perform exercise 3 times a week for 20 minutes each in the face of different barriers (range 0-100). Items are scored from 0-10 (Not confident to Very confident). ${ }^{21}$

Biomarkers-B-type natriuretic peptide (BNP) was analyzed on whole blood collected in EDTA using a commercially available Biosite Triage BNP Test point-of-service meter (fluorescence immunoassay). Serum BNP $>100 \mathrm{pg} / \mathrm{ml}$ supports a diagnosis of symptomatic heart failure.

Echocardiography-Serial echocardiographic studies were performed at baseline and at 12 weeks by a blinded assessor using a commercially available echocardiographic machine (GE Vivid 7). Assessments included mitral annular tissue Doppler measures, strain, strain rate, and flow propagation velocity. In particular, E/A (the ratio of mitral peak velocity of early filling to mitral peak velocity of late filling) and E/e' (the ratio of mitral peak velocity of early filling to early diastolic mitral annular velocity) were calculated to estimate degree of diastolic dysfunction. Image analysis was performed off-line using the Echopac digital system. Tapes were read by a single experienced cardiologist who was blinded to subject treatment assignment.

\section{Cardiorespiratory assessments during exercise}

At weeks 2 and 10 of training, all subjects underwent cardiorespiratory assessments for 5 minutes during each of three separate training phases. Mean $\mathrm{VO}_{2}$, respiratory rate and heart rate were assessed to provide a composite gauge of exercise intensity. In the tai chi group, VO2 was assessed during warm-up, meditation, and tai chi movement phases. In the aerobics group, $\mathrm{VO} 2$ was assessed during warm-up, cool-down and during the aerobic training phases. Gas exchange was assessed in-class at the community health facility using a MedGraphics ULTIMA CARDIO2 cart, and heart rate measurement was captured using a 
Marquette Electronics (Milwaukee, Wisconsin) series 8500 Holter monitor, digitized at 128 $\mathrm{Hz}$, and annotated using a MARS 8000 Holter scanner.

\section{Statistical analysis}

All statistical analyses were performed on an intention-to-treat basis. Two-sample Wilcoxon rank-sum tests were used to compare the distribution of change from baseline to 12 weeks between treatment and control groups for exercise and functional parameters, questionnaire scores, echocardiographic indices and BNP. Continuous data are summarized as mean (SD). Cardiorespiratory assessments during exercise are reported using descriptive statistics. Comparisons were also made between groups examining the pooled mean (not separated by phase or timepoint) for heart rate, percent maximal heart rate, respirations, oxygen consumption, and Borg rating using Wilcoxon-rank sum tests.

\section{Results}

\section{Baseline characteristics}

Characteristics of the 16 patients enrolled in the study are described in Table 2. Mean age $( \pm$ SD) of the study population was $66( \pm 12)$ years, and the distribution of New York Heart Association functional class (I, II, III) was 3,9, and 4 patients, respectively. Based on echocardiographic indices, seven patients had mild diastolic dysfunction (abnormal relaxation), six patients had moderate dysfunction (pseudo-normalization), and three patients had severe dysfunction (restrictive filling); seven patients exhibited left ventricular hypertophy. All patients were clinically euvolemic at baseline. The groups were similar with respect to demographics or co-morbidities except for physical activity and POMS score; members of the tai chi group reported more baseline activity and relatively worse moods. There were no differences in baseline exercise performance or quality-of-life scores.

\section{Feasibility, adherence, and safety}

We demonstrated feasibility of tai chi as an intervention for HFPEF. Patients were willing to be randomized. Mean class attendance rate was $89 \%$ for tai chi and $88 \%$ for aerobic exercise training. Data are unavailable with respect to specific frequency and duration of home practice. However, 7 out of 8 in the tai chi group and 4 out of 8 in the aerobics group selfreported compliance with home practice three times a week. No adverse events or hospitalizations occurred during class sessions or the study period. At a follow-up phone call conducted three months after the intervention ended, 6 out of 8 in the tai chi group reported continued practice of tai chi; two of these individuals reported starting aerobic training in addition to tai chi. Four out of 8 in the aerobics group reported continued aerobic training.

\section{Change in clinical outcomes after 12 weeks}

Tables 3, 4 and 5 present outcome measures at baseline and 12 weeks. Compared to those randomized to conventional aerobic exercise, subjects in tai chi showed no significant differences in peak $\mathrm{VO}_{2}$ but relatively greater improvements in $6 \mathrm{MW}$.

While both groups improved quality-of-life, there was no differential change between groups detected based upon MLHF score. However, the tai chi group showed improvements in the POMS-total mood disturbance and depression subscale.

There were increases in physical activity outside of classes as measured by the CHAMPS questionnaire in both groups, but no statistically significant difference between the groups. There were no significant changes in BNP in relation to exercise training. Echocardiographic indices of diastolic filling showed that E/e' improved after exercise in the aerobics group more so than tai chi, while left atrial size decreased in the tai chi group. 


\section{Characterization of tai chi and aerobic training intensity}

Table 6 shows the mean values of cardiorespiratory data collected during three phases of both tai chi and aerobic exercise sessions. Mean heart rate and $\mathrm{VO}_{2}$ were lower in tai chi. Respiratory rates were also slower in tai chi, particularly in the phase of training corresponding to meditation which deliberately incorporates slow, deep breathing.

\section{Discussion}

While HFPEF is common and associated with high morbidity and mortality, definitive treatment remains unclear. Exercise options that promote adherence are needed. Overall, this pilot study provides preliminary information regarding feasibility and potential safety of tai chi. Despite its relatively lower aerobic training intensity, tai chi may provide benefits that are similar to a typical conventional, community-based low-impact aerobics regimen in this population.

Within the context of this small study, we found that 6MW distance improved more significantly than with aerobic training. Likewise, depression scores decreased to a greater extent with tai chi. While preliminary, these findings are provocative. One possible physiological rationale is that as a training stimulus, tai chi places relatively more emphasis on skeletal muscle strengthening, stretching, and neuroregulatory enhancements that can translate into significant functional gains and which might be achieved rather efficiently using tai chi rather than conventional aerobic exercise techniques. While strength was not measured here, there are trials in the elderly that suggest that tai chi can enhance peripheral muscle strength (legs and hand grip), peripheral blood flow, and neuromuscular coordination (gross postural control and fine motor skills). $9,11,37,39-40$

Whereas Kitzman, et al. showed benefit of 16 weeks of aerobic training for patients with HFPEF, related analyses attributed peripheral differences in skeletal muscle to be a primary determinant of these training benefits. ${ }^{5}$ Aerobic training is associated with important peripheral advantages, particularly in regard to increased endothelial health and improved vasodilatory and perfusion capacities. However, strength and neuroautonomic physiologic effects also play important roles. These may be adequately achieved by other nonconventional exercise training modes. Consistently, guidelines for systolic heart failure call for strength training as part of a composite exercise regimen. ${ }^{22-24}$ In addition, intrinsic respiratory limitations have been recognized as an important component of HF pathophysiology with bearing on exercise intolerance, well-being, and prognosis. Inspiratory muscle training may provide key benefits, including improved inspiratory muscle and peripheral strength, functional capacity, ventilatory efficiency, perception of dyspnea and quality of life. ${ }^{25-26}$ Tai chi constitutes a unique blend of these exercise training attributes that may potentially address aerobic, strength, and even respiratory aspects of HFPEF. Importantly, studies of tai chi in varied populations have suggested good adherence and significant patient well-being. ${ }^{11}$

A related aspect of this study is that training intensity of tai chi appeared considerably lower than aerobic training while benefits were similar. Reduced oxygen consumption during tai chi training is likely related to the lower heart rate, since $\mathrm{VO}_{2}$ is based, in part, on heart rate responses. However, reduced breathing rate during tai chi suggests fundamentally different training effects compared to aerobic exercise.

Implications bear not only on the physiological gains facilitated by training, but the behavioral advantages of a lower cardiorespiratory training burden. Prior literature shows that exercise adherence and efficacy is more likely to be achieved with a regimen that can provide effective low intensity options, particularly as exercise is initiated, but that can also 
progress in intensity as tolerated or facilitate intermittent intervals of higher intensity. ${ }^{27-30}$ In this respect, tai chi, which is inherently less strenuous, but easily modified to tailor intensity, may directly address tolerability and accessibility, and thus, facilitate exercise adoption and maintenance long-term.

Safety implications may also be relevant. The slower heart rate and cardiovascular workload of tai chi as compared to aerobic exercise constitutes a more modest cardiovascular risk. The likelihood of significant adverse arrhythmia, ischemia, and/or other cardiovascular instability is proportionally reduced. Apparent safety with tai chi practice has been reported in prior trials in high-risk cardiovascular subjects, ${ }^{31-33}$ as well as other populations, including the transitionally frail, ${ }^{34}$ those with arthritis, ${ }^{35-36}$ and balance-impaired subjects with vestibular disease. ${ }^{37}$ Tai chi has also been proposed to be particularly suitable in older age, and thus among patients particularly predisposed to HFPEF. Optimal HFPEF exercise therapy must therefore address the fundamental pathophysiology of the disease itself, as well as the context of frailty and age-related limitations that are inherently associated with the disease.

Key limitations of the study are the small sample size and single recruitment site potentially affecting overall generalizability. This study, however, was designed to be a pilot, assess feasibility and gather preliminary estimates for a future trial. Nonetheless, because of the small sample, the likelihood of spurious findings may increase. In particular, we found that E/e' improved after aerobics, suggesting that aerobic training facilitated improved lucitropic function. While prior studies suggest that training benefits in both heart failure with preserved ejection fraction and with left ventricular dysfunction are mediated primarily by peripheral physiological adaptations rather than intrinsic cardiac effects, ${ }^{23}$ a recent trial by Edelmann did report improvements in diastolic function. ${ }^{38}$ It is also noted that the aerobic exercise group did not significantly improve peak VO2 after 12 weeks, despite our cardiorespiratory data suggesting that adequate training should have been achieved. Limitations in aerobic training efficacy in heart failure have been described, and it is possible that these are amplified in older adults, specifically with HFPEF with a typical community-based intervention.

Despite the prevalence of HFPEF, clinical studies in this population have been notoriously challenging as many confounding issues (e.g., chronic pulmonary disease, and normal agerelated ventricular diastolic filling changes) complicate identification of an ideal study population. Albeit a small sample, our study included clinical, echocardiographic and serological parameters to assess patients with HFPEF. To our knowledge, this is the first study to demonstrate the applicability of tai chi to a HFPEF population and to characterize training intensity as compared to aerobic exercise in this population. In summary, our data suggest that tai chi may achieve exercise training benefits with improved 6MW that are similar to a typical community-based low-impact aerobic exercise in patients with HFPEF. Benefits were seen with tai chi at a lower training intensity that may potentially facilitate safety and longer-term exercise adherence. As heart failure is a pervasive and rapidly growing medical problem, our data provide intriguing rationale for further study of tai chi exercise in this cardiac population.

\section{Acknowledgments}

Grant support: This study was supported by an award from the National Center for Complementary and Alternative Medicine (Yeh, K23 AT00002624) and in part by the Beth Israel Deaconess Medical Center General Clinical Research Center grant (RR 01032) from the NIH. 


\section{REFERENCES}

1. Kaila K, Haykowsky MJ, Thompson RB, Ian Paterson D. Heart failure with preserved ejection fraction in the elderly: scope of the problem. Heart Fail Rev. Aug 5.2011

2. Kitzman DW, Brubaker PH, Morgan TM, Stewart KP, Little WC. Exercise training in older patients with heart failure and preserved ejection fraction: a randomized, controlled, single-blind trial. Circ Heart Fail. Nov 1; 2010 3(6):659-67. Epub 2010 Sep 17. [PubMed: 20852060]

3. O'Connor CM, Whellan DJ, Lee KL, Keteyian SJ, Cooper LS, Ellis SJ, Leifer ES, Kraus WE, Kitzman DW, Blumenthal JA, Rendall DS, Miller NH, Fleg JL, Schulman KA, McKelvie RS, Zannad F, Piña IL. HF-ACTION Investigators. Efficacy and safety of exercise training in patients with chronic heart failure: HF-ACTION randomized controlled trial. JAMA. Apr 8; 2009 301(14): 1439-50. [PubMed: 19351941]

4. Tierney S, Mamas M, Skelton D, Woods S, Rutter MK, Gibson M, Neyses L, Deaton C. What can we learn from patients with heart failure about exercise adherence? A systematic review of qualitative papers. Health Psychology. 2011; 30(4):401-410. [PubMed: 21534681]

5. Haykowsky MJ, Brubaker PH, John JM, Stewart KP, Morgan TM, Kitzman DW. Determinants of exercise intolerance in elderly heart failure patients with preserved ejection fraction. $\mathrm{J}$ Am Coll Cardiol. Jul 12; 2011 58(3):265-74. [PubMed: 21737017]

6. Cheng, MC. Master Cheng's Thirteen Chapters on T'ai Chi Chuan. Sweet Chi Press; New York: 1982.

7. Wayne PM, Kaptchuk TJ. Challenges inherent to t'ai chi research: part I--t'ai chi as a complex multicomponent intervention. J Altern Complement Med. 2008; 14(1):95-102. [PubMed: 18199021]

8. Lan C, Lai JS, Chen SY. Tai Chi Chuan: an ancient wisdom on exercise and health promotion. Sports Med. 2002; 32(4):217-24. [PubMed: 11929351]

9. Wu G. Evaluation of the effectiveness of Tai Chi for improving balance and preventing falls in the older population--a review. J Am Geriatr Soc. 2002; 50:746-754. [PubMed: 11982679]

10. Li JX, Hong Y, Chan KM. Tai chi: physiological characteristics and beneficial effects on health. Br J Sports Med. 2001; 35(3):148-56. [PubMed: 11375872]

11. Wang C, Collet JP, Lau J. The effect of Tai Chi on health outcomes in patients with chronic conditions: a systematic review. Arch Intern Med. 2004; 164(5):493-501. [PubMed: 15006825]

12. Yeh GY, Wood MJ, Lorell BH, et al. Effects of tai chi mind-body movement therapy on functional status and exercise capacity in patients with chronic heart failure: a randomized controlled trial. Am J Med. 2004; 117(8):541-8. [PubMed: 15465501]

13. Yeh GY, McCarthy EP, Wayne PM, Stevenson LW, Wood MJ, Forman D, Davis RB, Phillips RS. Tai chi exercise in patients with chronic heart failure: a randomized clinical trial. Arch Intern Med. Apr 25; 2011 171(8):750-7. [PubMed: 21518942]

14. The American Thoracic Society. ATS statement: guidelines for the six-minute walk test. Am J Respir Crit Care Med . 2002; 166:111-117. [PubMed: 12091180]

15. Kao W, Jessup M. Exercise testing and exercise training in patients with congestive heart failure. J Heart Lung Transplant. 1994; 13:S117-121. [PubMed: 7947867]

16. Podsiadlo D, Richardson S. The timed "Up \& Go": a test of basic functional mobility for frail elderly persons. J Am Geriatr Soc. 1991; 39(2):142-8. [PubMed: 1991946]

17. Stewart AL, Mills KM, King AC, Haskell WL, Gillis D, Ritter PL. CHAMPS physical activity questionnaire for older adults: outcomes for interventions. Med Sci Sports Exerc. Jul; 2001 33(7): 1126-41. [PubMed: 11445760]

18. Rector T, Tschumperlin L, Kubo S, et al. Use of the Living with Heart Failure questionnaire to ascertain patients' perspectives on improvement in quality of life versus risk of drug-induced death. J Card Fail. 1:201-206. [PubMed: 9420652]

19. McNair, DM.; Lorr, M.; Droppelman, LF. Manual for the profile of mood states. EDITS Educational and Industrial Testing Services, Inc.; San Diego: 1992.

20. Wang C, Bannuru R, Ramel J, Kupelnick B, Scott T, Schmid CH. Tai Chi on psychological wellbeing: systematic review and meta-analysis. BMC Complement Altern Med. 2010; 21:10, 23. 
21. Resnick B, Jenkins LS. Testing the reliability and validity of the Self-Efficacy for Exercise scale. Nurs Res. May-Jun;2000 49(3):154-9. [PubMed: 10882320]

22. Wilson JR, Mancini DM, Dunkman WB. Exertional fatigute due to skeletal muscle dysfunction in patients with heart failure. Circulation. 1993; 87:470-5. [PubMed: 8425294]

23. Esposito F, Mathieu-Costello O, Shabetai R, Wagner PD, Richardson RS. Limited maximal exercise capacity in patients with chronic heart failure; partitioning the contributors. JACC. 2010; 55:1945-54. [PubMed: 20430267]

24. Borlag BA, Olson TP, Lam CS. Global cardiovascular reserve dysfunction in heart failure with preserved ejection fraction. JACC. 2010; 56:845-54. [PubMed: 20813282]

25. Dall'Ago P, Chiappa GR, Guths H, Stein R, Ribeiro JP. Inspiratory muscle training in patients with heart failure and inspiratory muscle weakness: a randomized trial. J Am Coll Cardiol. Feb 21.2006 47(4):757. [PubMed: 16487841]

26. Wong E, Selig S, Hare DL. Respiratory muscle dysfunction and training in chronic heart failure. Heart Lung Circ. May; 2011 20(5):289-94. [PubMed: 21435947]

27. Cress ME, Buchner DM, Prohaska T, Rimmer J, Brown M, Macera C, Dipietro L, Chodzko-Zajko W. Best practices for physical activity programs and behavior counseling in older adult populations. J Aging Phys Act. Jan; 2005 13(1):61-74. [PubMed: 15677836]

28. Belardinelli R, Lacalaprice F, Ventrella C, Volpe L, Faccenda E. Waltz dancing in patients with chronic heart failure: new form of exercise training. Circ Heart Fail. Jul; 2008 1(2):107-14. [PubMed: 19808280]

29. Downing J, Balady GJ. The role of exercise training in heart failure. J Am Coll Cardiol. Aug 2; 2011 58(6):561-9. [PubMed: 21798416]

30. Wisloff U, Stoylen A, Loennechen JP, et al. Superior cardiovascular effect of aerobic interval training versus moderate continuous training in heart failure patients. Circulation. 2007; 115:3086-94. [PubMed: 17548726]

31. Lan C, Chen SY, Wong MK, Lai JS. Tai chi training for patients with coronary heart disease. Med Sport Sci. 2008; 52:182-94. [PubMed: 18487898]

32. Channer K, Barrow D, Barrow R, Osborne M, Ives G. Changes in haemodynamic parameters following Tai Chi Chuan and aerobic exercise in patients recovering from acute myocardial infarction. Postgrad Med J. 1996; 72:349-351. [PubMed: 8758013]

33. Lan C, Chen S, Lai J, Wong M. The effect of Tai Chi on cardiorespiratory function in patients with coronary artery bypass surgery. Med Sci Sports Exerc. 1999; 31:634-638. [PubMed: 10331880]

34. Wolf SL, Sattin RW, Kutner M, O'Grady M, Greenspan AI, Gregor RJ. Intense Tai Chi exercise training and fall occurrences in older, transitionally frail adults: a randomized, controlled trial. $\mathrm{J}$ Am Geri Soc. 2003; 51:1693-1701.

35. Wang C, Schmid CH, Hibberd PL, et al. Tai Chi is effective in treating knee osteoarthritis: a randomized controlled trial. Arthritis Rheum. 2009; 61(11):1545-53. [PubMed: 19877092]

36. Wang C. Tai Chi improves pain and functional status in adults with rheumatoid arthritis: results of a pilot single-blinded randomized controlled trial. Med Sport Sci. 2008; 52:218-29. [PubMed: 18487901]

37. Wayne PM, Krebs DE, Wolf SL, et al. Can Tai Chi improve vestibulopathic postural control? Arch Phys Med Rehabil. 2004; 85(1):142-52. [PubMed: 14970982]

38. Edelmann F, Gelbrich G, Dungen HD, et al. Exercise training improves exercise capacity and diastolic function in patients with heart failure with preserved ejection fraction. JACC. 2011; 58(17):1780-91. [PubMed: 21996391]

39. Fong SM, Ng GY. The effects on sensorimotor performance and balance with Tai Chi training. Arch Phys Med Rehabil. 2006; 87:82-87. [PubMed: 16401443]

40. Xu DQ, Li JX, Hong Y. Effect of regular Tai Chi and jogging exercise on neuromuscular reaction in older people. Age Ageing. 2005; 34:439-444. [PubMed: 15919758]

41. American College of Sports Medicine. ACSM's Guidelines for Exercise Testing and Prescription. Lippincott Williams \& Wilkins; Philadelphia, Pa: 2010. p. 286-299. 


\section{Table 1}

Description of the tai chi intervention

\begin{tabular}{|c|c|c|}
\hline Week & Activities & Approx. Duration (min) \\
\hline \multirow[t]{6}{*}{1} & Introductory Session: Overview of Program & \\
\hline & 1. Tai Chi principles, philosophies & 15 \\
\hline & 2. Demonstration of Tai Chi form & 10 \\
\hline & 3. Expectations of participants & 10 \\
\hline & 4. Description of class format & 5 \\
\hline & 5. Participation in warm-up exercises & 30 \\
\hline \multirow[t]{17}{*}{$2-5$} & Warm-up Exercises (Repeated during all sessions) & \\
\hline & 1. Standing: & \\
\hline & a) "Drumming the body" & 6 \\
\hline & b) "Swinging to connect kidney and lungs" & 3 \\
\hline & c) "Washing the body with qi" & 3 \\
\hline & d) Standing meditation and breathing & 3 \\
\hline & 2. Sitting: & \\
\hline & a) Neck/Shoulder stretches & 6 \\
\hline & b) Arm/Leg stretches & 3 \\
\hline & c) Sitting meditation and breathing & 6 \\
\hline & Total Warm-up time & 30 \\
\hline & Tai Chi Movements & \\
\hline & 1. "Raising the Power" & $5-10$ \\
\hline & 2. "Withdraw and Push" & 5 per side \\
\hline & (Warm-up and Movements 1-2) & \\
\hline & 3. "Grasp Sparrows Tail" & 5 per side \\
\hline & 4. "Brush Knee Twist Step" & 5 per side \\
\hline \multirow[t]{2}{*}{$10-12$} & (Warm-up and Movements 1-4) & \\
\hline & 5. "Wave Hands Like Clouds" & $5-10$ \\
\hline
\end{tabular}


Table 2

Baseline characteristics of the study sample

\begin{tabular}{|c|c|c|}
\hline & Tai Chi (n=8) & Aerobic Exercise (n=8) \\
\hline \multicolumn{3}{|l|}{ Demographic Factors: } \\
\hline Age (years $\pm \mathrm{SD})$ & $68 \pm 11$ & $63 \pm 11$ \\
\hline Sex: Men & $4(50)$ & $4(50)$ \\
\hline \multicolumn{3}{|l|}{ Race: } \\
\hline Black & $0(0)$ & $3(37)$ \\
\hline White & $8(100)$ & $5(62)$ \\
\hline \multicolumn{3}{|l|}{ Clinical Classification: } \\
\hline Body Weight (kg) & $87 \pm 32$ & $94 \pm 35$ \\
\hline Body Mass Index (kg/m2) & $32 \pm 10$ & $34 \pm 14$ \\
\hline Systolic BP (mmHg) & $135 \pm 10$ & $134 \pm 19$ \\
\hline Diastolic BP (mmHg) & $74 \pm 10$ & $68 \pm 11$ \\
\hline Heart Rate (bpm) & $64 \pm 10$ & $68 \pm 11$ \\
\hline NYHA Class I & $1(12)$ & $2(25)$ \\
\hline Class II & $4(50)$ & $5(63)$ \\
\hline Class III & $3(38)$ & $1(12)$ \\
\hline Weekly caloric expenditure of reported activities $(\mathrm{kcal} / \mathrm{wk}){ }^{* *}$ & $2.4 \pm 1.9$ & $1.3 \pm 2.3$ \\
\hline \multicolumn{3}{|l|}{ Physical Capacity: } \\
\hline Peak VO2 (ml/kg/min) & $14.5 \pm 7$ & $13.1 \pm 5$ \\
\hline Peak RER & $1.15 \pm 0.08$ & $1.15 \pm 0.14$ \\
\hline Exercise time (min) & $7.2 \pm 6.3$ & $6.6 \pm 2.8$ \\
\hline $6 \mathrm{MW}(\mathrm{m})$ & $335.4 \pm 174$ & $349.7 \pm 216$ \\
\hline \multicolumn{3}{|l|}{ Medications: } \\
\hline ACE inhibitor/ARB & $6(75)$ & $4(50)$ \\
\hline Digoxin & $0(0)$ & $0(0)$ \\
\hline Diuretics & $6(75)$ & $6(75)$ \\
\hline Beta-blockers & $6(75)$ & $5(62)$ \\
\hline Calcium antagonists & $2(25)$ & $5(62)$ \\
\hline Nitrates & $1(12)$ & $1(12)$ \\
\hline \multicolumn{3}{|l|}{ Self-Reported Co-Morbidities: } \\
\hline Coronary artery disease & $3(37)$ & $3(37)$ \\
\hline Arrhythmia & $2(25)$ & $3(37)$ \\
\hline Hypertension & $5(62)$ & $7(87)$ \\
\hline Diabetes & $2(25)$ & $1(12)$ \\
\hline High cholesterol & $4(50)$ & $4(50)$ \\
\hline Asthma & $1(12)$ & $3(37)$ \\
\hline Anxiety & $4(50)$ & $1(12)$ \\
\hline Depression & $3(37)$ & $3(37)$ \\
\hline Arthritis & $2(25)$ & $3(37)$ \\
\hline Cancer & $3(37)$ & $1(12)$ \\
\hline
\end{tabular}




\begin{tabular}{|lcc|}
\hline & Tai Chi $(\mathbf{n}=\mathbf{8})$ & Aerobic Exercise $(\mathbf{n}=\mathbf{8})$ \\
Renal disease & $3(37)$ & $3(37)$ \\
\hline
\end{tabular}

Data are presented as mean $\pm \mathrm{SD}$, or $\mathrm{N}(\%)$

**

As measured by the CHAMPS questionnaire 


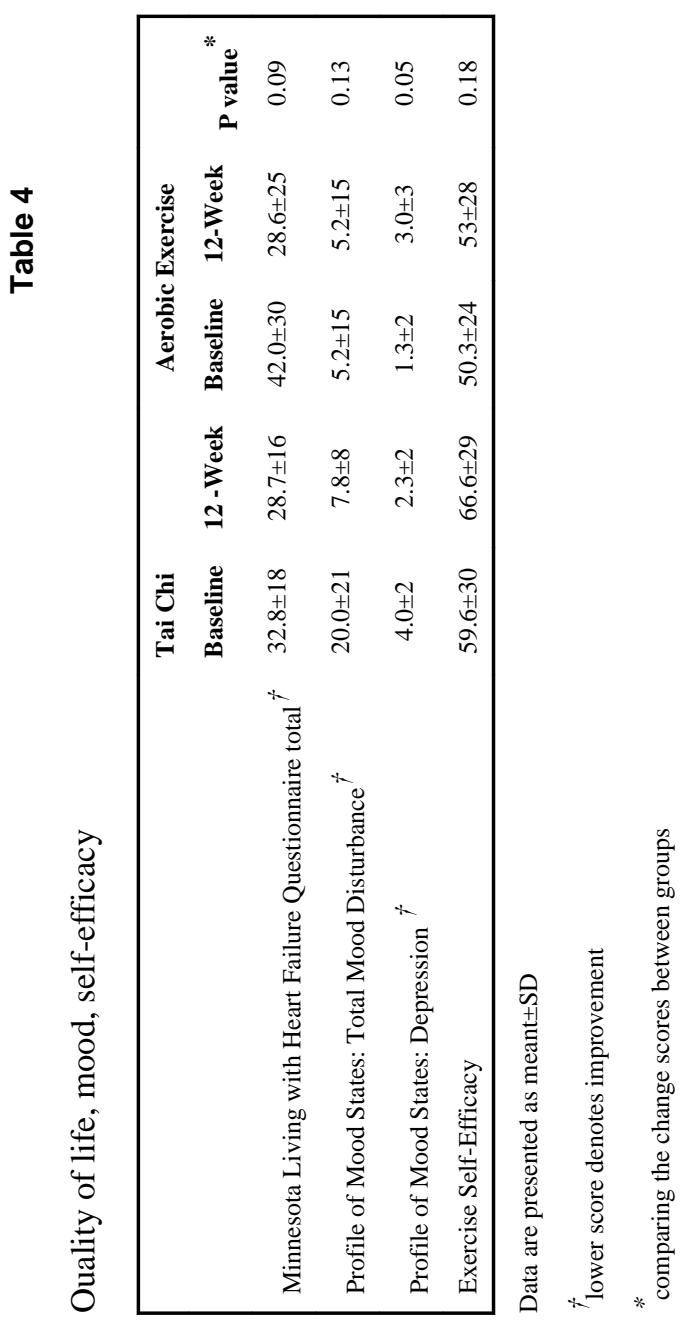

Congest Heart Fail. Author manuscript; available in PMC 2014 March 01. 


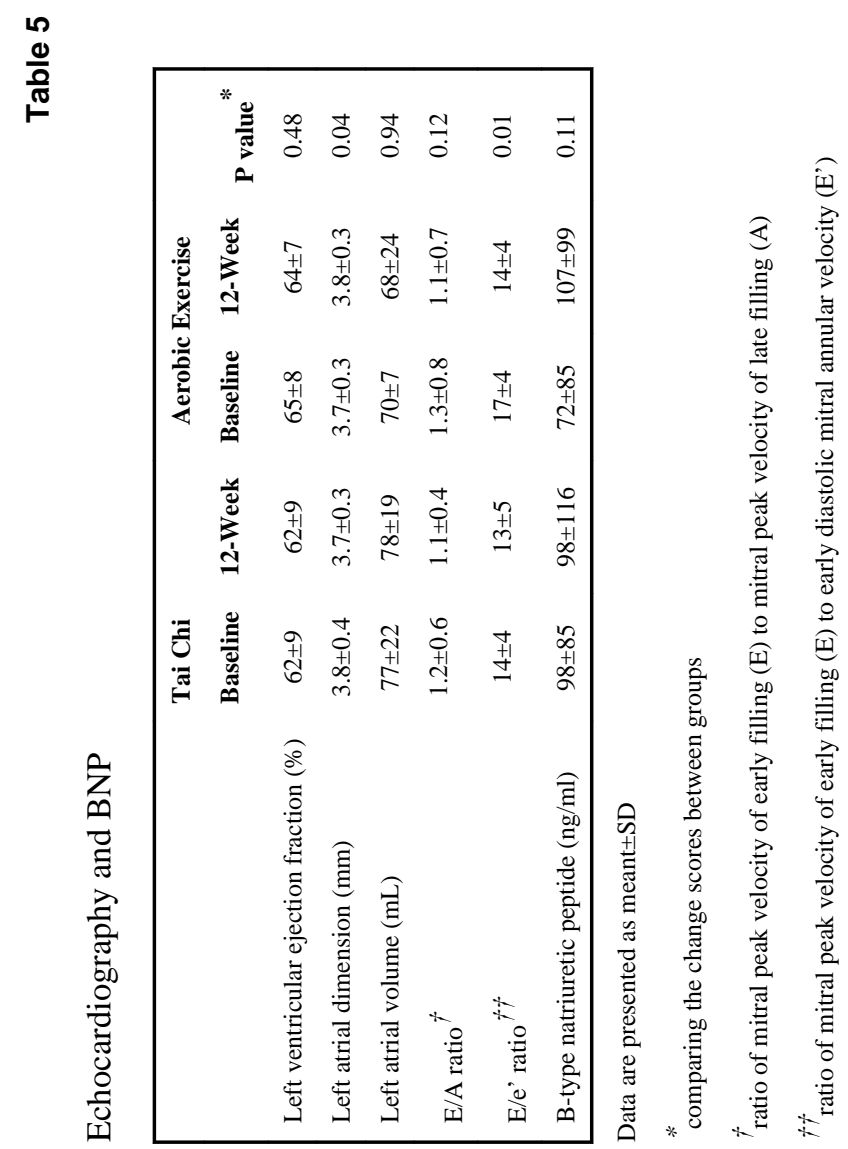

Congest Heart Fail. Author manuscript; available in PMC 2014 March 01. 


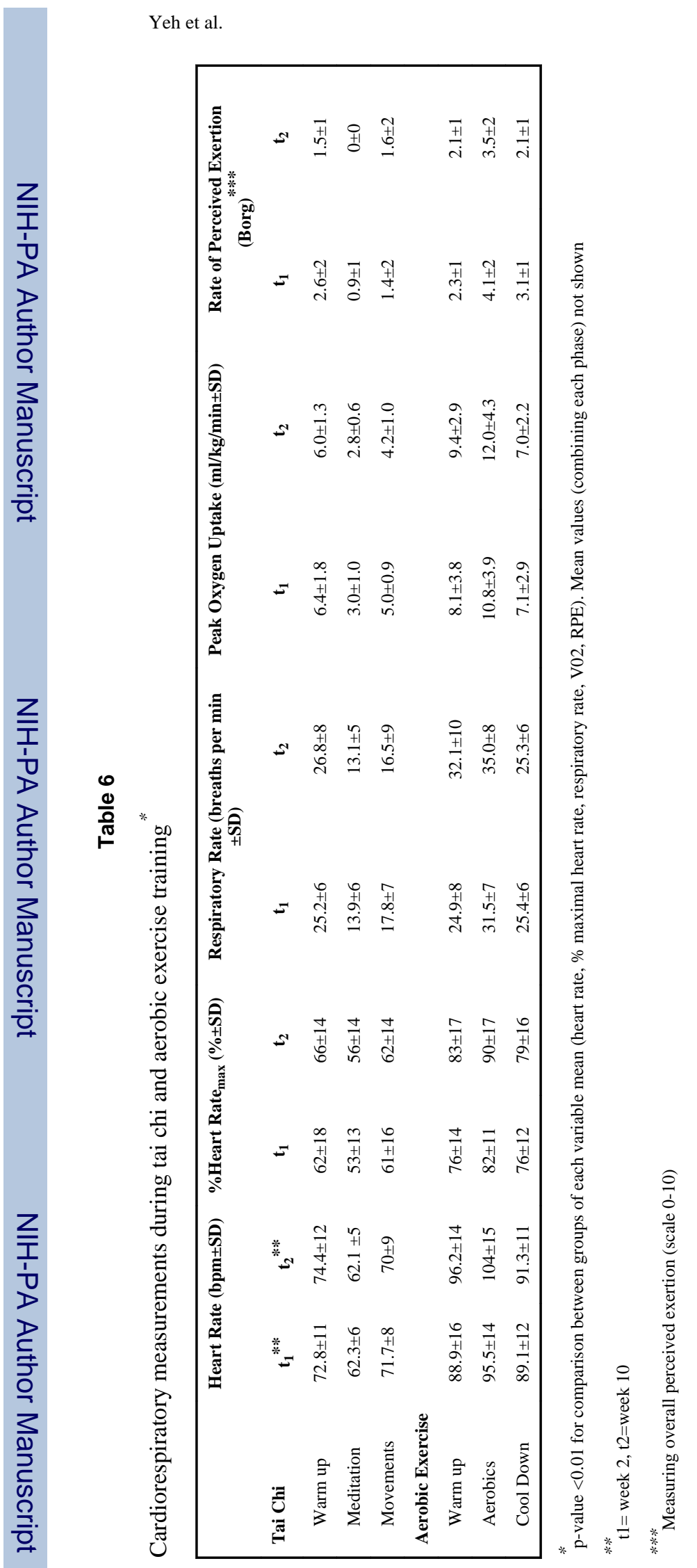

Congest Heart Fail. Author manuscript; available in PMC 2014 March 01. 